\title{
Detection of X-ray emission from $\beta$ Pictoris with XMM-Newton: a cool corona, a boundary layer or what?
}

\author{
M. Hempel ${ }^{1,2}$, J. Robrade ${ }^{2}$, J.-U. Ness ${ }^{2,3}$, and J. H. M. M. Schmitt ${ }^{2}$ \\ 1 AIU Jena, Schillergäßchen 2-3, 07745 Jena, Germany \\ e-mail: marc@astro.uni-jena.de \\ 2 Hamburger Sternwarte, Gojenbergsweg 112, 21029 Hamburg, Germany \\ 3 Department of Physics, Rudolf Peierls Centre for Theoretical Physics, University of Oxford, 1 Keble Road, \\ Oxford OX13NP, UK
}

Received 22 December 2004 / Accepted 9 May 2005

\begin{abstract}
Pictoris (HR 2020) is the most prominent prototype of stars with circumstellar disks and has generated particular interest in the framework of young planetary systems. Given its spectral type A5, stellar activity is not expected. Nevertheless, resonance lines of $\mathrm{C} \mathrm{III} \mathrm{and} \mathrm{O} \mathrm{VI} \mathrm{typical} \mathrm{for} \mathrm{a} \mathrm{chromosphere} \mathrm{and} \mathrm{transition} \mathrm{region} \mathrm{have} \mathrm{been} \mathrm{unambiguously} \mathrm{detected} \mathrm{with}$ FUSE. We present results from an XMM-Newton observation of $\beta$ Pic and find evidence for X-ray emission. In particular, we detected an emission of O VII at $21.6 \AA$ with the MOS detectors. These findings present a challenge for the development of both stellar activity and disk models. We discuss and investigate various models to explain the observed emission including the presence of a cool corona and a boundary layer.
\end{abstract}

Key words. stars: activity - stars: circumstellar matter - stars: individual: HR 2020 - ultraviolet: stars X-rays: stars

\section{Introduction}

The A5 star $\beta$ Pictoris (HR 2020) is well-known for its circumstellar (CS) debris disk (Smith \& Terrile 1984) seen nearly edge-on. Spectroscopic observations exhibit conspicuous and highly variable narrow absorption lines interpreted as falling evaporating bodies (FEB, Ferlet et al. 1987): kilometre-sized cometary-like objects on star-grazing orbits pass near $\beta$ Pic and evaporate gas leading to imprints up to $v_{\mathrm{K}} \approx 400 \mathrm{~km} \mathrm{~s}^{-1}$ in the photospheric spectrum. Among other stars like Vega and $\alpha$ PsA, CS disks have generated particular interest in the framework of young planetary systems. Although numerous stars possessing CS material have been found so far, $\beta$ Pic is the most prominent prototype of stars with a CS disk and thus $\beta$ Pic presents a challenge for the development of theoretical disk models (see e.g. Kamp et al. 2003). In this context it is necessary to determine the temperature structure of disks in order to obtain chemical compositions and disk masses. Since the chemistry in CS disks is driven by the stellar radiation field, such models require a realistic stellar flux as input. Upper atmospheric layers like chromospheres will have a pronounced effect on disks since the chromospheric UV photons lead to warmer disks due to an increase in the heating rate. The same applies to the presence of a X-ray radiation field from a possible stellar corona. Depending on the spectral type of the host star it is thus necessary to account for the chromospheric UV and coronal X-ray flux. For the case of HR 4796A/B, consisting of an A0V-type star and a $\mathrm{M}$ dwarf companion, this has been carried out by Chen \& Kamp (2004) in their study of the CS environment of this source. Similar to $\beta$ Pic, HR 4796A harbours CS material which has been successfully imaged (Schneider et al. 1999). From the companion's ROSAT X-ray flux of $F_{\mathrm{X}} \lesssim 2 \times 10^{-4} \mathrm{erg} / \mathrm{cm}^{2} / \mathrm{s}$ at the position of HR 4796 , Chen \& Kamp (2004) estimated that X-ray heating due to the M dwarf is insignificant for the case of HR 4796A/B.

In general, A-type stars are not considered to be chromospheric and coronal stars. Radiation hydrodynamic model calculations show that they do not possess significant outer convection zones (Freytag et al. 1996) and therefore one does not expect chromospheric and coronal X-ray emission from these stars. Yet, small turbulent velocities are present in A-type stars. Holweger et al. (1997) determined the microturbulence of $\beta$ Pic to be $\xi_{\mathrm{t}}=4 \pm 0.3 \mathrm{~km} \mathrm{~s}^{-1}$, a value slightly higher than what is generally expected for A stars.

In order to study the possible occurrence of chromospheres and transition regions in A-type stars Simon et al. (2002) have carried out FUSE observations in the C III at 977/1175 and OVI at 1032/1037 ̊ lines for a sample of seven stars in the temperature range between $T_{\text {eff }} \sim 7800 \mathrm{~K}$ and $8600 \mathrm{~K}$. They find an abrupt drop of the chromospheric emission in a very narrow temperature range of just $\pm 50 \mathrm{~K}$, centered around $T_{\text {eff }} \approx 8250 \mathrm{~K}$. In the sample of stars analyzed by Simon et al. (2002) the A4 V star $\tau^{3}$ Eri is the hottest $\left(T_{\text {eff }}=8210 \mathrm{~K}\right)$ 
Table 1. Observation log of $\beta$ Pic.

\begin{tabular}{llllll}
\hline \hline \multirow{2}{*}{ Instrument } & \multirow{2}{*}{ Filter } & Mode & Duration $(\mathrm{s})$ & Observation time & Good times (ks) \\
\hline MOS1 & Thick & Full Frame & 73668 & 2004-01-04 18:52:18-2004-01-05 15:20:06 & 72 \\
MOS2 & Thick & Full Frame & 73677 & 2004-01-04 18:52:13-2004-01-05 15:20:10 & 72 \\
PN & Medium & Full Frame & 72036 & 2004-01-04 19:14:34-2004-01-05 15:15:10 & 55 \\
RGS1 & & Spectroscopy & 73912 & 2004-01-04 18:51:30-2004-01-05 15:23:22 & - \\
RGS2 & & Spectroscopy & 73913 & 2004-01-04 18:51:35-2004-01-05 15:23:28 & - \\
\hline
\end{tabular}

main-sequence star known to date to show evidence of hot material in a chromosphere and transition region. However, X-ray emission from $\tau^{3}$ Eri has so far not been detected; it shows up neither as a source in the ROSAT all-sky survey nor was it detected in Einstein Observatory pointed observations (cf., Schmitt et al. 1990). Interestingly, the effective temperature of $\tau^{3}$ Eri is similar to that of $\beta$ Pic $\left(T_{\text {eff }}=8200 \mathrm{~K}\right.$, Holweger et al. 1997). The steep decline of the boundary line for convection as found by Simon et al. (2002) is consistent with recent stellar structure models (Christensen-Dalsgaard 2000; Kupka \& Montgomery 2002).

The expected absence of X-ray emission from A-type stars is verified from ROSAT all-sky survey observations, which show a much lower detection rate for A-type stars as compared to F- or G-type stars (cf., Schmitt \& Liefke 2004). In the X-ray picture, the "earliest" late-type star showing magnetic activity as evidenced through X-ray emission is the nearby, single A7V star Altair with $L_{X}=6.4 \times 10^{27} \mathrm{erg} / \mathrm{s}$ (Hünsch et al. 1998).

Nevertheless some A-type stars do show significant X-ray emission (Hünsch et al. 1999). These X-ray detections are usually ascribed to unidentified late-type companions, although it is difficult to rule out an intrinsic X-ray flux of the A-type stars. Support for this scenario has been derived by Schmitt \& Kürster (1993), who observed the eclipsing binary system $\alpha$ CrB with ROSAT, an eclipsing binary system consisting of an A0 V and a G5 V star. The X-ray light curve of the system shows a total X-ray eclipse during secondary optical minimum, with the $G$ star behind the A star. The totality of the eclipse demonstrates that - as "expected" - the A-type component in $\alpha \mathrm{CrB}$ is $\mathrm{X}$-ray dark and that the X-ray flux arises essentially exclusively from the late-type companion. Furthermore, Chung et al. (2004) detected Doppler shifts in the eclipsing binary Algol, consisting of a B8 V primary and a K2 IV secondary, ascribed to the orbital motion of the K2 star Algol B. Their Chandra HETGS observations thus demonstrate that the X-ray emission of Algol is dominated by the K2 secondary while the early-type primary component is X-ray faint and most likely $\mathrm{X}$-ray dark.

In the case of $\beta$ Pic no convincing evidence of X-ray emission or any other signature of magnetic activity from the star itself has been found so far. However, a study based on FUSE spectra by Deleuil et al. (2001) led to the surprising result that $\beta$ Pic may actually show signatures of stellar activity. The FUSE observations obtained by Deleuil et al. (2001) show pronounced emission lines of C III at 977.020/1175.711 $\AA$ and of O VI at 1031.926/1037.617 $\AA$, respectively. Such highly ionized species are a typical signature of the presence of a chromosphere-transition region complex, which consequently led Bouret et al. (2002) to the development of a magnetic activity scenario for $\beta$ Pic on the basis of their FUSE spectra.

Starting from the FUSE detection of O VI emission lines from $\beta$ Pic, it is natural to also expect emission lines from $\mathrm{O}$ VII, which can be conveniently diagnosed with XMM-Newton. We therefore carried out a deep X-ray observation with XMM-Newton in order to determine the X-ray emission level from $\beta$ Pic especially in this line and the purpose of this paper is the presentation of this observation and a discussion of our results.

\section{Observations, data analysis and results}

On 2004 January 4 we observed $\beta$ Pic for $73.6 \mathrm{ks}$ with XMMNewton; details of the observation are given in Table 1 .

Data were collected with all X-ray detectors onboard XMM-Newton, the EPIC (European Photon Imaging Camera) and RGS (Reflection Grating Spectrometer) detectors. A detailed description of the XMM-Newton instruments can be found in Ehle et al. (2003). The XMM-Newton data reduction was carried out using the standard XMM-Newton Science Analysis System (SAS) routines, version 5.4.1 and standard selection criteria were applied for filtering the data. The EPIC detectors were operated with two different filters, the PN camera with the medium filter, the two MOS cameras with the thick filter. The PN observation is more strongly affected by proton contamination, which results in a total good time of $55 \mathrm{ks}$ for the PN camera while the MOS observations provide a good time of $72 \mathrm{ks}$ each. Analysis of EPIC data was performed in the energy range $0.2-12.0 \mathrm{keV}$ for the MOS and $0.2-15.0 \mathrm{keV}$ for the PN detector. In the RGS no useful signal could be detected from $\beta$ Pic, therefore we restricted our analysis to the EPIC data.

\subsection{XMM-Newton EPIC PN results}

In the EPIC PN image a source can be clearly identified at the precise position of $\beta$ Pic; extracting the photons with their individually measured energies we constructed an X-ray spectrum as plotted in Fig. 1. 216 source photons were extracted from a circular region with a $20^{\prime \prime}$ radius around the nominal position of $\beta$ Pic. For the spectrum, 723 background photons were extracted from a four times larger background region on the same CCD. The EPIC PN spectrum clearly shows the typical spectral shape of optical contamination of the CCD chips (see, e.g., Ness et al. 2004), and therefore no claim of a X-ray detection can be made on the basis of the EPIC PN observation alone. 


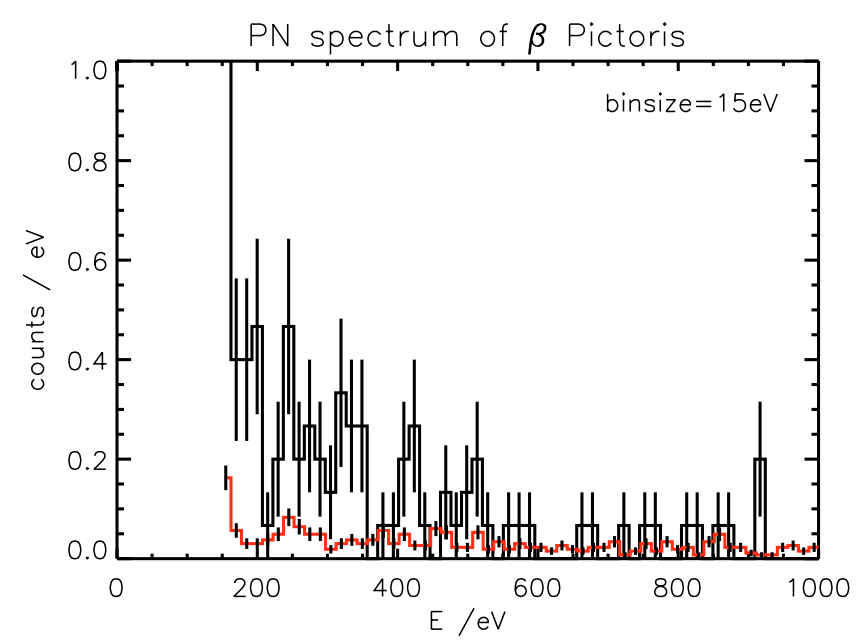

Fig. 1. The PN spectrum of $\beta$ Pic applying a binsize of $15 \mathrm{eV}$ (black, upper curve) and the background level (red/grey, lower curve). Note the significant difference of both histograms in the range between $200 \mathrm{eV}$ and $500 \mathrm{eV}$.

\subsection{XMM-Newton EPIC MOS results}

Due to the fact that, on the one hand, the FUV observations of $\beta$ Pic with FUSE (Deleuil et al. 2001) clearly detected OVI emission, but on the other hand, the EPIC PN data show no clear X-ray signal, we specifically searched for emission from OVII in the EPIC MOS data. Hot plasma in the temperature range $5.6<\log T[\mathrm{~K}]<6.1$ produces strong OVII emission lines in the He-like triplet at wavelengths of $21.6 \AA, 21.8 \AA$ and $22.1 \AA$, respectively, corresponding to energies of $574 \mathrm{eV}$, $569 \mathrm{eV}$, and $561 \mathrm{eV}$ with very little continuum emission. Since the individual triplet lines cannot be resolved in the EPIC MOS detectors with their medium spectral resolution, we searched for photons from this triplet in a narrow energy band centered around $565 \mathrm{eV} \pm 100 \mathrm{eV}$, selected a priori to contain basically all triplet photons. A circular source extraction region with a $20^{\prime \prime}$ radius around the nominal position of $\beta$ Pic was chosen. In order to determine a reliable background value we extracted photons from various source free regions on the central CCD of the MOS detectors; the total area chosen for background determination is 111 times larger than the source region. The same exercise was also carried out for other energy bands below and above the energy interval centered on the OVII triplet energies in order to search for possible additional source signals and to check our background estimation procedures. The results of these extraction procedures are summarized in Table 2, where we list for a total of 6 energy bands (the band $0.465-0.665 \mathrm{keV}$ is centered on the OVII triplet) the extracted source cell counts for MOS1, MOS2 and their sum, the (scaled) extracted background values and their sum, the ratio between source cell and background counts, and the Poisson probabilities for obtaining the extracted source counts (or higher), assuming only a Poisson signal from the expected background, again for MOS1 and MOS2 separately and their combined probability.

Inspection of Table 2 shows that in five of the six considered energy bands the ratio between source and background counts is very close to unity and that the probabilities of obtaining the observed number of counts are always 0.1 or higher.
However, in the a priori chosen energy band $0.465-0.665 \mathrm{keV}$ an excess is observed both for MOS1 (7 counts observed, 2.5 expected) and MOS2 (10 counts observed, 3.6 expected) and of course for both detectors combined (17 counts observed, 6.1 expected). While the probabilities for obtaining the individual MOS 1 and MOS 2 counts by chance are already low $(0.014$ and 0.004 , respectively), the combined probability for the assumption of observing background fluctuations both in MOS1 and MOS2, is only $5.7 \times 10^{-5}$. This small number combined with the fact that in the other energy bands no significant detections are obtained makes us confident that our background estimates are reliable and that the observed excess in the $0.465-$ $0.665 \mathrm{keV}$ band is real (with a formal statistical confidence of 0.999943 ) despite the low overall number of recorded counts.

Interpreting the observed 10.9 excess photons as a true source signal, we can convert this number into an energy flux. With an effective area in the considered energy band (465$665 \mathrm{eV}$ ) of $86 \mathrm{~cm}^{2}$ for each of the two detectors, an encircled energy fraction of $\sim 75 \%$ for the used source cell regions and the CCD live time of $72 \mathrm{ks}$, we can calculate the recorded energy flux in the OVII-triplet. While other X-ray lines (e.g. Hlike NVII at $500 \mathrm{eV}$ ) and continuum emission may contribute to the observed flux, OVII is clearly the major contributor in this energy band, unless extremely unusual abundance patterns are assumed. In this fashion we arrive at an observed energy flux of $6.0 \times 10^{-16} \mathrm{erg} / \mathrm{cm}^{2} / \mathrm{s}$ in the OVII triplet; if one were to choose not to interpret these counts as a true source signal or to assume other relevant contributors in this energy band, this number would have to be treated as an upper limit.

\subsection{ROSAT HRI results}

After carrying out the XMM-Newton observation we analyzed an (apparently) unpublished ROSAT HRI observation of $\beta$ Pic obtained in February 1996 (ROR number WG202151). Like the EPIC PN image, the ROSAT HRI image shows a clear source at the position of $\beta$ Pic. Again the source is seen mainly in the lower pulse height channels suggesting a UV origin of the observed signal. Restricting the analysis to HRI pulse height channels $2-8$, we find a count rate of $1.41 \times$ $10^{-3} \mathrm{cts} / \mathrm{s}$. Using a $U$ band magnitude of 4.13 and the ROSAT HRI UV contamination calibration curve derived by Berghöfer et al. (1999) we calculate an expected contaminating ROSAT HRI flux of $4.3 \times 10^{-4} \mathrm{cts} / \mathrm{s}$, which lies considerably below the actually observed value. However, it is obviously quite courageous to interpret the formal excess as a true X-ray signal since the calibration curve by Berghöfer et al. (1999) has been derived from a rather small number of stars.

\section{Discussion}

\subsection{Internal consistency of $X$-ray results}

Accepting the MOS data as a X-ray detection, we derive an observed X-ray count rate of $1.5 \times 10^{-4} \mathrm{cts} / \mathrm{s}$ for $\beta$ Pic. This is clearly inconsistent with the excess with respect to the expected flux from UV contamination as formally derived from the ROSAT HRI data; we therefore conclude that all of the 
Table 2. The photons of $\beta$ Pic. The a priori chosen energy band between $0.465 \mathrm{keV}$ and $0.665 \mathrm{keV}$ is centered on the OvII triplet at $21.6 \AA / 21.8 \AA / 22.1 \AA$ A. Note the significantly different ratio between source and background counts as well as the conspicuously lower probabilities for the assumption of observing background fluctuations in this energy band.

\begin{tabular}{l|ccc|ccc|c|ccc}
\hline \hline & \multicolumn{3}{|c|}{ Source region } & \multicolumn{3}{|c|}{ Scaled background } & \multicolumn{3}{c|}{ SRC/BG } & \multicolumn{3}{c}{ Probability } \\
\hline Energy (keV) & MOS1 & MOS2 & $\Sigma$ & MOS1 & MOS2 & $\Sigma$ & Ratio & PPM1 & PPM2 & PPM1/2 \\
\hline $0.2-0.465$ & 4 & 7 & 11 & 3.9 & 7.0 & 10.9 & 1.0 & 0.55 & 0.55 & 0.30 \\
$0.465-0.665$ & 7 & 10 & 17 & 2.5 & 3.6 & 6.1 & 2.8 & 0.014 & 0.004 & $5.7 \times 10^{-5}$ \\
$0.665-1.0$ & 6 & 2 & 8 & 3.5 & 3.7 & 7.2 & 1.1 & 0.14 & 0.88 & 0.13 \\
$1.0-2.0$ & 19 & 11 & 30 & 14.3 & 15.2 & 29.5 & 1.0 & 0.13 & 0.89 & 0.12 \\
$2.0-5.0$ & 15 & 9 & 24 & 11.9 & 11.4 & 23.3 & 1.0 & 0.22 & 0.80 & 0.18 \\
$5.0-12.0$ & 13 & 16 & 29 & 19.1 & 18.3 & 37.4 & 0.8 & 0.94 & 0.74 & 0.69 \\
\hline
\end{tabular}

Table 3. Comparison of O VI and OvII fluxes. The last column contains the X-ray fluxes calculated by Hünsch et al. (1998) (besides AB Dor). All data are given in $10^{-14} \mathrm{erg} / \mathrm{cm}^{2} / \mathrm{s}$. See Sect. 3.2 for details.

\begin{tabular}{|c|c|c|c|c|c|c|c|}
\hline Star & HD & Sp.-Type & O VI $(1032 \AA)$ & \multicolumn{3}{|c|}{ O VII $(r+i+f)$} & $f_{\mathrm{x} 14}$ \\
\hline & & & & LETG & MEG & RGS1 & \\
\hline Procyon & 61421 & F5 IV-V & $223 \pm 49^{a}$ & $66 \pm 7$ & - & - & 1293.9 \\
\hline$\alpha$ Cen A & 128620 & $\mathrm{G} 2 \mathrm{~V}$ & $95.4 \pm 9.5^{a}$ & $21 \pm 5$ & - & - & $990.0^{c}$ \\
\hline$\alpha$ Cen B & 128621 & $\mathrm{~K} 1 \mathrm{~V}$ & $61.6 \pm 11.5^{a}$ & $25 \pm 6$ & - & $84 \pm 29$ & $990.0^{c}$ \\
\hline AB Dor & 36705 & K1 III p & $44.9 \pm 4.5^{a}$ & - & $32 \pm 13$ & $49 \pm 7$ & $5100^{d}$ \\
\hline$\epsilon$ Eri & 22049 & $\mathrm{~K} 2 \mathrm{~V}$ & $43.3 \pm 4.3^{a}$ & $71 \pm 8$ & - & $64 \pm 16$ & 1687.0 \\
\hline AU Mic & 197481 & M0 V & $20.9 \pm 2.4^{a}$ & - & $32 \pm 13$ & $67 \pm 8$ & - \\
\hline Altair & 187642 & A7 V & $82.1 \pm 8.2^{a}$ & - & - & - & 201.3 \\
\hline$\beta$ Pic & 39060 & A5 V & $5.5^{b}$ & - & - & $<0.3$ & - \\
\hline
\end{tabular}

${ }^{a}$ Redfield et al. (2002), ${ }^{b}$ Deleuil et al. (2001), ${ }^{c}$ combined flux of $\alpha$ Cen A and $\alpha$ Cen B,

${ }^{d}$ derived using $7 \mathrm{cts} / \mathrm{s}$ (Kürster et al. 1997).

ROSAT HRI data are due to UV contamination from $\beta$ Pic's photosphere. On the other hand, performing the same procedure for the EPIC PN camera as carried out and described in Sect. 2.2 for the MOS data, we find in the energy band 0.465$0.665 \mathrm{keV}$ a total of 25 source counts where 12 background counts are expected. Considering the different characteristics of the instrument and shorter effective exposure time one expects approximately 16 source counts in the PN detector. The net number of 13 source counts is thus consistent with the total number of counts detected in the two MOS cameras. We note that optical/UV contamination dominates at low energies, and our measurements above $0.665 \mathrm{keV}$ are fully compatible with being background. If one therefore attributes the detected EPIC PN counts below $0.465 \mathrm{keV}$ to optical/UV contamination and the recorded ROSAT HRI counts to UV contamination, all the obtained X-ray measurements are internally consistent.

\subsection{Comparison to other cool stars}

It is instructive to compare the obtained FUV and X-ray data to those of other cool stars. In Table 3, we list FUSE measurements of the OvI line at $1031.926 \AA$ together with various measurements of the OVII triplet obtained with Chandra and XMM-Newton as well as broad band measurements obtained with the ROSAT PSPC. The OVI data are taken from Redfield et al. (2002) and Deleuil et al. (2001). The OvII fluxes were measured by us using CORA (Ness \& Wichmann 2002); for details see Ness et al. (2004). The RGS data are included for completeness only. Since we did not detect a useful RGS signal for $\beta$ Pic due to the small number of photons, this value has to be considered as an upper limit. The ROSAT measurements are from the compilation by Hünsch et al. (1998); the AB Dor PSPC flux is based on $7 \mathrm{cts} / \mathrm{s}$ (Kürster et al. 1997), converted to fluxes using the HEASARC tool PIMMS $(0.1-2 \mathrm{keV})$. We perform this comparison for the inactive stars Procyon, $\alpha$ Cen A and B and the "early" late-type star Altair, as well as the active stars $\epsilon$ Eri, AB Dor, and AU Mic. Note that for Altair no high resolution spectroscopic X-ray measurements are available. From Table 3 it is clear that for all stars (possibly except Altair) the OVI and OVII flux is of the same order of magnitude, in contrast to $\beta$ Pic. This suggests a fundamental difference between the stars with outer convection zones and $\beta$ Pic.

\subsection{A cool corona?}

If we attribute the OVI line detection obtained with FUSE and the OVII X-ray detection obtained with XMM-Newton to a stationary corona, we can obtain a temperature and emission measure estimate for such a structure. Using the CHIANTI code (Dere et al. 1997) to compute the flux ratios between the overall OVII triplet (i.e., the sum of resonance, intercombination and forbidden lines) and the OVI doublet (i.e., the sum of the $1032 \AA$ and $1037 \AA$ lines) as a function of temperature, we can compare the measurements for various stars (cf. Table 3) with 


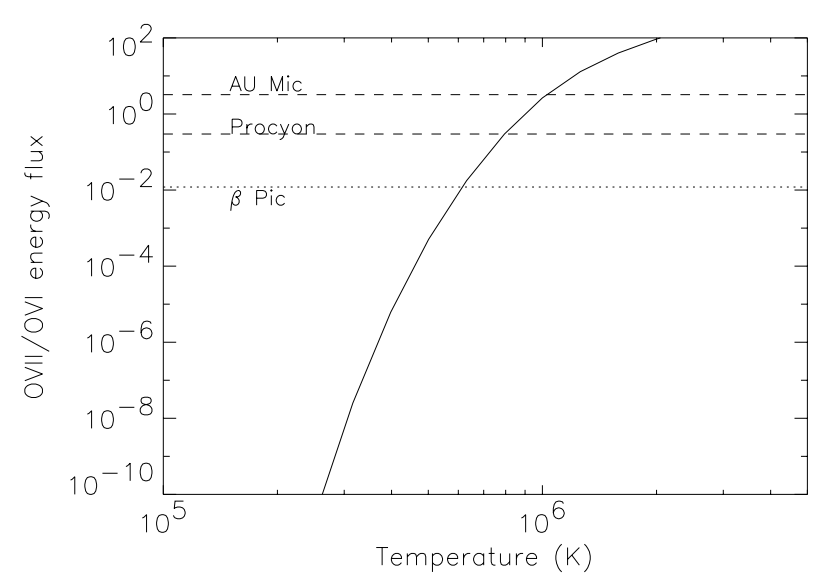

Fig. 2. Flux ratio of the OvII triplet vs. OvI doublet. See Table 3 for the corresponding OVII and OVI fluxes. For $\beta$ Pic, we used the OvII flux of $6.0 \times 10^{-16} \mathrm{erg} / \mathrm{cm}^{2} / \mathrm{s}$ derived in Sect. 2.2.

theoretical expectations in Fig. 2. As is obvious from Fig. 2, the thus derived temperature of $\approx 600000 \mathrm{~K}$ is far lower than that of any comparison star. Accepting for the moment this value, we require volume emission measures of $\approx 10^{52} \mathrm{~cm}^{-3}$ to account for both the FUSE OVI and XMM-Newton OVII measurements. Naturally, these emission measure values depend very sensitively on the adopted temperatures, and choosing higher temperatures leads to significantly lower emission measures. Based on the X-ray detection of Altair (Golub et al. 1983; Schmitt et al. 1985; Freire Ferrero et al. 1995), and regarding the similarity between $\beta$ Pic and Altair, Bouret et al. (2002) suspected that a warm corona such as that of Altair (Freire Ferrero et al. 1995) can be expected for $\beta$ Pic as well.

\subsection{Comparison of FUSE line profiles}

In order to address the issue of whether there is a connection between the FUV and X-ray emissions obtained for the A5V star $\beta$ Pic and the A7 V star Altair (HR 7557), we retrieved the archival FUSE data analysed by Deleuil et al. (2001) and compared them with spectra of the same lines from Altair ${ }^{1}$.

Figures 3 and 4 show the overlaid emission line profiles of C III at $977.020 \AA$ and O VI at $1031.926 \AA$ for both $\beta$ Pic and Altair, respectively. As can be seen from Figs. 3 and 4 , the emission lines from $\beta$ Pic are clearly broader than those of Altair. This is remarkable since the significantly different projected rotational velocities of both stars would suggest exactly the opposite: while $\beta$ Pic rotates with $v \sin i_{\beta \text { Pic }}=132 \mathrm{~km} \mathrm{~s}^{-1}$ (Holweger et al. 1999), Altair apparently rotates much faster with $v \sin i_{\text {Altair }}=208 \mathrm{~km} \mathrm{~s}^{-1}$ (Holweger et al. 1999). Therefore one would expect the profiles of Altair to be far broader than those of $\beta$ Pic. Figures 3 and 4 show the contrary. This strongly suggests that for $\beta$ Pic an additional line broadening mechanism must exist which exceeds the rotational line broadening and is not present in the otherwise similar star Altair. We note in this context that "suprarotational" line broadening is often encountered in studies of emission lines originating from chromospheres and/or transition regions. For example,

\footnotetext{
1 Dataset IDs Q1190103000 ( $\beta$ Pic) and P1180701000 (Altair).
}

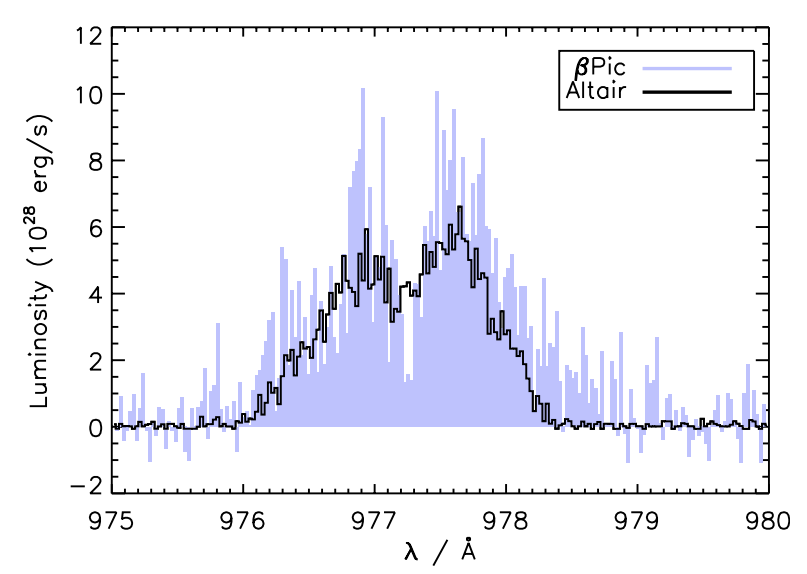

Fig. 3. Comparison of spectra from $\beta$ Pic (blue/grey) and Altair (black) obtained from the FUSE archive. The profiles show the CIII $977 \AA$ Aresonance line. Note that despite the significantly lower rotational velocity $\left(v \sin i_{\beta \text { Pic }}=132 \mathrm{~km} \mathrm{~s}^{-1}, v \sin i_{\text {Altair }}=208 \mathrm{~km} \mathrm{~s}^{-1}\right)$ the profile of $\beta$ Pic is at least as broad as Altair's.

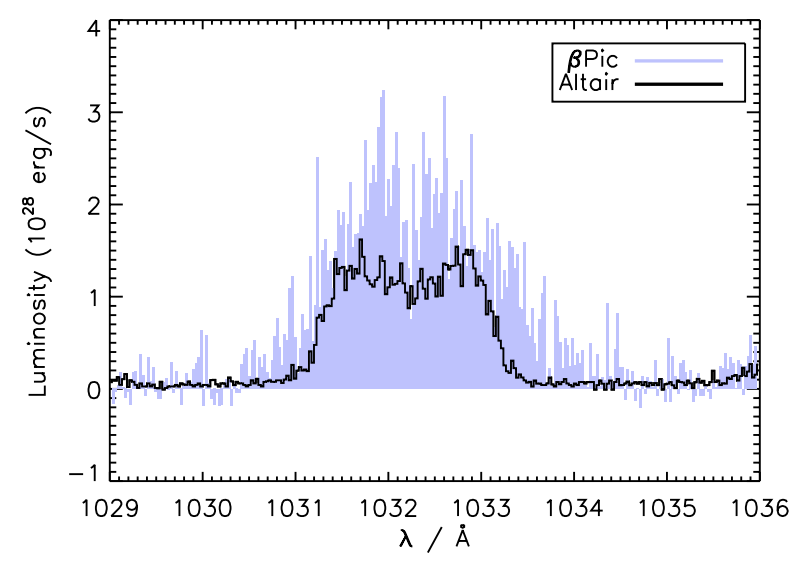

Fig. 4. OVI at $1032 \AA$ shows that the profile obtained for $\beta$ Pic is broader than the one of Altair. As for CIII at $977 \AA$ we consider this as an effect from the infalling material.

Ayres et al. (1997) measured such broadening in their study of moderate-mass giant stars, which, however, are slow rotators compared to the A-stars considered in this paper; their Fig. 9 shows the dependence of the nonthermal broadening on $v \sin i$ for four stars, suggesting a correlation between excess broadening and stellar rotation, and the authors then attribute this broadening to a thick transition zone extending to $h \sim R_{\star}$. As far as main-sequence stars of earlier spectral type are concerned, Simon \& Landsman (1997), using HST GHRS data, and Simon et al. (2002), using FUSE data, describe the emission line profiles of C III $\lambda$ 977, O VI $\lambda 1032$, and Si III $\lambda 1206$ from $\tau^{3}$ Eri, $\alpha$ Cep, and Altair as significantly broader than what one would expect from the $v \sin i$ values, without, however, giving a quantitative analysis. In their FUSE study of $\beta$ Pic, Bouret et al. (2002) suggest that the suprarotational broadening can be explained in terms of an extended coronal magnetosphere which traps and heats the gas in the corotating lobes. If the chromospheric and transition region line broadening in $\tau^{3}$ Eri is indeed comparable to that observed in Altair as suggested by Simon \& Landsman (1997), $\tau^{3}$ Eri with $v \sin i=120 \mathrm{~km} \mathrm{~s}^{-1}$ would in fact be quite similar to $\beta$ Pic. 
While no disk is known around $\tau^{3}$ Eri, both Ohanesyan (1997) and Song et al. (2001) argue for the presence of a disk around $\tau^{3}$ Eri, suggesting $\tau^{3}$ Eri to be "Vega-like" based on IRAS excesses at at $12 \mu \mathrm{m}$ and $25 \mu \mathrm{m}$ (rather than at $60 \mu \mathrm{m}$ ).

\subsection{A boundary layer?}

Are there other origins for the additional line broadening mechanism required to explain the OVI line profile observed in $\beta$ Pic? Let us recall that material from its circumstellar debris disk is falling onto $\beta$ Pic with a more or less constant rate. Narrow circumstellar absorption components in the core of the Ca II K line are a well-studied phenomenon: the star shows a seemingly stable central absorption and a variable component which may vanish at times. The variability in the core of the Ca II $\mathrm{K}$ line on short timescales is attributed to the above mentioned falling evaporating bodies (FEB's, Ferlet 1987, see Sect. 1). Petterson \& Tobin (1999) carried out a long-term study on the spectral variability of $\beta$ Pic in $\mathrm{Ca}$ II $\mathrm{H}$ and $\mathrm{K}$ between 1994 and 1996 and inferred event rates of 400 per year for high velocity features (HVF's), while low velocity features are seemingly more stable and may even persist for several nights. In our study of high-resolution spectra $(R \approx 217000)$ we have detected nightly variations in the Ca II $\mathrm{K}$ line of $\beta$ Pic (Hempel \& Schmitt 2003). Brandeker et al. (2004) analyzed the spatial structure of the $\beta$ Pic gas disk in a distance range from the star from $13 \mathrm{AU}$ out to $323 \mathrm{AU}$ and found 88 spatially resolved emission lines in their UVES spectra. From the velocity shift of the NE and SW side observed in the $\mathrm{Na}_{2}$ emission they deduced a Keplerian rotation of the outer disk. It is thus clear that the disk around $\beta$ Pic contains not only debris and dust, but also gas.

Let us therefore hypothesize that $\beta$ Pic is still an accretion disk now, which it must have been in the very early days of $\beta$ Pic's existence. Of course, radiation pressure acts - as always - against accretion, however, according to Lagrange et al. (1998, Table 4), the radiation pressure would only prevent some ions, but not the bulk of the material from accretion; also, the source of viscosity, which would be required for angular momentum transport, remains unclear.

Let us first consider the accretion rate necessary to account for the observed high-temperature plasma. Modelling the emitting gas as an isothermal, optically thin gas cloud of temperature $T$, the FUSE and XMM-Newton observations determine the volume emission measure $V E M=n_{\mathrm{e}}^{2} \times V$. The emitting volume $V$ is assumed to have a height $h$ much smaller than the stellar radius $R$, so that $V=4 \pi R^{2} h f$, where $f$ is the unknown filling factor of the accreting material. The hot gas is assumed to predominantly cool through radiation, and the radiative cooling time scale $\tau_{\text {rad }}$ is given by $\tau_{\text {rad }}=\frac{3 k T}{2 n_{\mathrm{e}} P(T)}$, where $P(T)$ denotes the radiative cooling function of the plasma as a whole. As the gas cools, it is accreted and settles onto the star. Assuming explicitly that radiative cooling is the dominant energy loss mechanism, the radial accretion velocity $v_{\text {rad }}$ is given by $v_{\text {rad }}=\frac{h}{\tau_{\text {rad }}}$. The overall mass accretion rate then becomes $\dot{M}_{\text {acc }}=4 \pi R^{2} f \rho v_{\text {rad }}$, where $\rho$ denotes the density of the X-ray emitting layers. Since $\rho=\mu m_{\mathrm{H}} n_{\mathrm{e}}$, we finally obtain

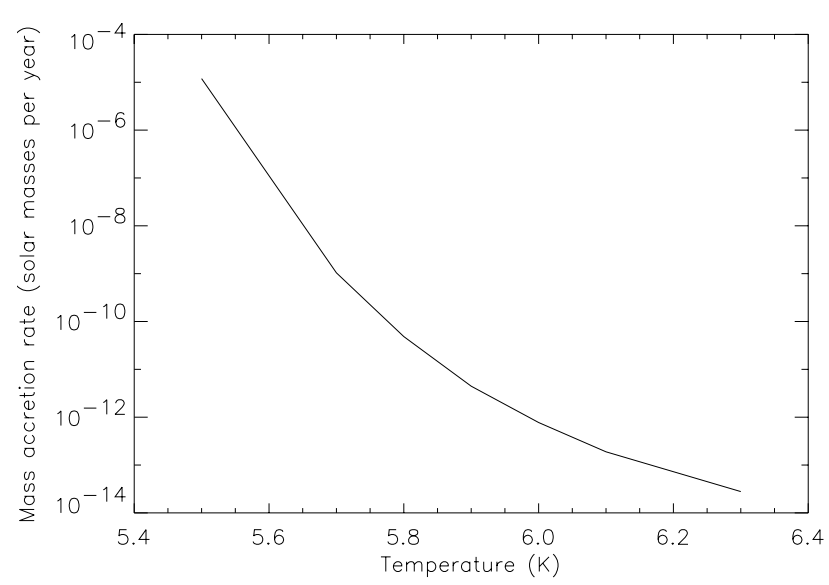

Fig. 5. Mass accretion rates vs. temperature. See Sect. 3.5 for details.

$\dot{M}_{\text {acc }}=\frac{8 \pi f R^{2} \mu m_{\mathrm{H}} n_{\mathrm{e}} h n_{\mathrm{e}} P(T)}{3 k T}=\frac{2 V E M \mu m_{\mathrm{H}} P(T)}{3 k T}$, i.e., an expression that depends only on measured or (more or less) known quantities. Unfortunately, the emission measure VEM, derived from the UV and X-ray data, depends very sensitively on the adopted temperature, and also, the plasma cooling function $P(T)$ depends on temperature and on the elemental abundances in the hypothesized accretion stream. In particular the latter may be far from the cosmic abundance and in fact only oxygen is observed. With the above formulae and the observed VEM we can compute $\dot{M}_{\text {acc }}$ as a function of temperature (see Fig. 5) assuming cosmic abundances (Holweger 2001), an ideal gas, and a plasma cooling function $P(T)$ as computed with the CHIANTI package (Dere et al. 1997). Since temperatures between $5.8<\log T<6.0$ are the most likely ones, we conclude that accretion rates between $10^{-10}-10^{-12} M_{\odot} \mathrm{yr}^{-1}$ would be required to explain the observed emissions via a boundary layer. Assuming reasonable values for $\beta$ Pic's mass, radius, and luminosity, $M=1.75 M_{\odot}, R=1.53 R_{\odot}, \log \left(L / L_{\odot}\right)=0.95$ (Di Folco et al. 2004), the resulting accretion luminosities $L_{\text {acc }}=\frac{G \dot{M}_{\text {acc }} M}{R}$ would then be in the range of $10^{31}-10^{29} \mathrm{erg} / \mathrm{s}$, i.e., only a fraction of $10^{-3}-10^{-5}$ of $\beta$ Pic's luminosity and therefore very difficult to detect. Holweger et al. (1997) have performed a detailed abundance analysis of $\beta$ Pic and found it to be consistent with solar abundance; this result would exclude mass accretion rates $>10^{-13} M_{\odot} \mathrm{yr}^{-1}$ consisting of material with depleted elemental abundances, while material with solar-like abundances could be accreted at much higher rates. King \& Patten (1992) analyzed accretion scenarios in $\beta$ Pic in the context of the $\lambda$ Boo phenomenon and estimated $1.5 \times 10^{-19} \leq \dot{M}_{\mathrm{FEB}} \leq 2 \times 10^{-14} M_{\odot} \mathrm{yr}^{-1}$ for the accretion due to FEBs and $7.5 \times 10^{-19} \leq \dot{M}_{\mathrm{CS}} \leq 6 \times 10^{-10} M_{\odot} \mathrm{yr}^{-1}$ for an accretion of CS material onto $\beta$ Pic. Unaffected by the fact that a metal-deficiency of $\beta$ Pic could not be established in an abundance analysis (Holweger et al. 1997, see also Gerbaldi et al. 1993) and thus the $\lambda$ Boo nature of $\beta$ Pic could not be confirmed, the estimated accretion rate is consistent with our values and we therefore conclude that at present there are no strong arguments against an accretion onto $\beta$ Pic with mass accretion rates in the range $10^{-10}-10^{-12} M_{\odot} \mathrm{yr}^{-1}$.

How is the accretion disk material actually accreted onto the star? This may occur by free fall from some inner truncation 
radius of the disk resulting in velocities of up to $400 \mathrm{~km} \mathrm{~s}^{-1}$, which are in fact obtained and observed for FEBs. However, line broadening of $400 \mathrm{~km} \mathrm{~s}^{-1}$ is certainly larger than what is required to explain the broadening observed in the FUSE spectra. Alternatively, we may assume a Keplerian disk reaching down almost to the stellar surface and releasing its energy in a narrow boundary layer. Assuming reasonable values for $\beta$ Pic's mass and radius, $M=1.75 M_{\odot}$ and $R=$ $1.53 R_{\odot}$ (Di Folco et al. 2004), we calculate a Keplerian velocity of $v_{\mathrm{K}} \approx 470 \mathrm{~km} \mathrm{~s}^{-1}$ directly at its surface. On the other hand, since the inclination angle is very close to $90^{\circ}$, we expect an equatorial rotation velocity $v_{\mathrm{eq}} \approx 132 \mathrm{~km} \mathrm{~s}^{-1}$ (Holweger et al. 1999). The kinetic energy contained in this velocity difference $\Delta V=v_{\mathrm{K}}-v_{\mathrm{eq}} \approx 340 \mathrm{~km} \mathrm{~s}^{-1}$ is available for dissipation and the value of $\Delta V$ should be a strict upper limit for the additionally occurring line broadening.

The kinetic energy of the incoming material is dissipated in a thin turbulent boundary layer. Applying the turbulent CroccoBusemann relations (White 1991) we find the following relation between boundary layer temperature $T$ and velocity $u$ :

$T=T_{\text {photo }}+\left(T_{\text {acc }}+\frac{\Delta V^{2}}{2 c_{\mathrm{p}}}\right) \frac{u}{\Delta V}-\frac{u^{2}}{2 c_{\mathrm{p}}} ;$

here $T_{\text {photo }}$ and $T_{\text {acc }}$ denote the temperatures of the photosphere and accretion disk respectively, $\Delta V$ the velocity difference between surface and Keplerian velocity and $c_{\mathrm{p}}$ the specific heat. In Fig. 6 we plot the temperature vs. velocity for accreting material onto $\beta$ Pic in a boundary layer. It can be seen that a maximum of about $T_{\max } \approx 3.5 \times 10^{5} \mathrm{~K}$ is reached for material accreting with $v \approx 170 \mathrm{~km} \mathrm{~s}^{-1}$. A temperature of about $350000 \mathrm{~K}$, in turn, corresponds to the line formation temperature of the observed emissions of CIII and O VI. At face value this is a factor of two lower, but considering the simplicity of the model the hypothesis of accretion of circumstellar material in a boundary layer is at least in principle capable of explaining the presence of CIII and O VI and the weakness of O VII without the ad hoc assumption of a chromosphere. For a more sophisticated model, detailed hydrodynamical calculations would be needed. Note that this scenario is also applicable to explain the additional broadening mechanism of the observed CIII and O VI emissions.

\subsection{Implications for disk models}

We compared $\beta$ Pic's broadened highly ionized resonance lines of C III and O VI analyzed by Deleuil et al. (2001) to the same lines of Altair. Our results show that the emission from the CIII and OVI lines are significantly broadened compared to the otherwise similar star Altair. Furthermore, we found X-ray emission from $\beta$ Pic in the OVII triplet at $21.6 \AA$. We have shown that our findings are consistent with the existence of either a cool corona or with the accretion of circumstellar material in a boundary layer without the ad hoc assumption of a chromosphere. These results present a challenge for the development of stellar/coronal models but one further point compared to, e.g. the active A7 V star Altair, is important especially for the case of $\beta$ Pic: the star harbours a well-known circumstellar debris disk with a diameter of approximately $1000 \mathrm{AU}$. Thus $\beta$ Pic

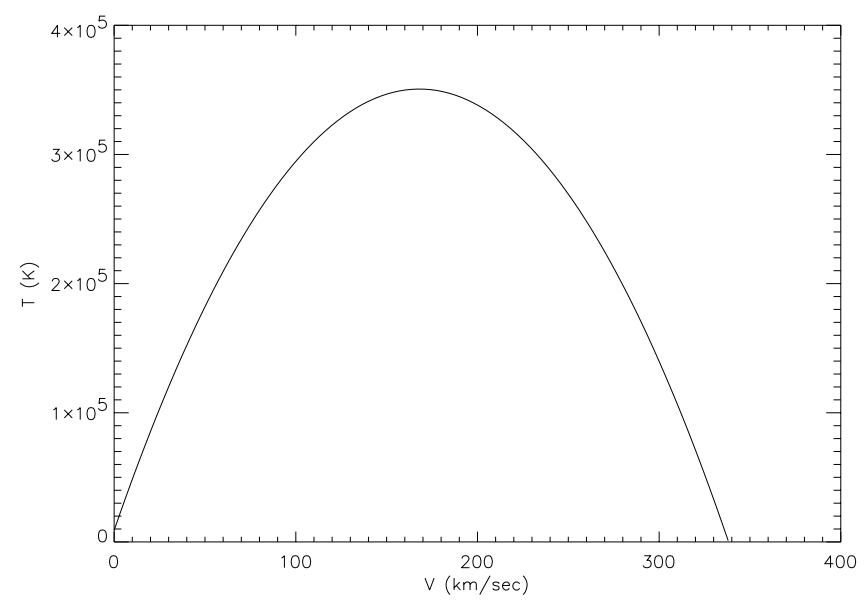

Fig. 6. Temperature of accreted material in a boundary layer according to the turbulent Crocco-Busemann relations (White 1991); see Sect. 3.5 and Eq. (1) for details. For infalling material with $v \approx$ $170 \mathrm{~km} \mathrm{~s}^{-1}$ we find a temperature of about $350000 \mathrm{~K}$ which is sufficient for the formation of CIII and OVI.

serves as a prototype: one important result of the IRAS and ISO missions is the detection of many IR-excess stars with CS disks and rings of gas and dust. One vital question still under debate is how to determine the masses of these disks. CS disk masses are an important ingredient in assessing the formation process of planets - either via core-accretion or gravitational instabilities. Therefore it is mandatory to develop models to describe the chemical structure of CS disks. So far, such models have often neglected the existence of chromospheres - even in the case of late-type stars. Nevertheless, it is known that the stellar radiation field drives the chemistry in CS disks and that the solar ultraviolet ionizing flux weakens with time. Thus the influence of the stellar UV field on CS disks is expected to be greater for younger stars. One attempt to include the chromosphere of late-type stars has been made by Kamp \& Sammar (2004), who studied the influence of the chromosphere of a G5 star on the chemistry in the late phases of disk evolution. Their calculations show that in the inner disk $(\$ 400 \mathrm{AU})$ the chromospheric $\mathrm{UV}$ emission is the driving mechanism in chemistry and that differences in the $\mathrm{H}$ and $\mathrm{CO}$ abundances by more than a factor of 10 occur if one neglects the presence of a chromosphere. Given the fact that highly ionized species were detected in the FUSE spectra of $\beta$ Pic, these processes may also have a pronounced effect on its disk chemistry. Since the calculations by Kamp \& Sammar (2004) were carried out for a G star, it would be desirable to extend the disk models to A-type stars. The model by Kamp \& Sammar (2004) does not consider an X-ray flux. Although the nature of the detected X-ray emission from $\beta$ Pic is not fully clear yet it is most likely that the $\mathrm{X}$-ray flux will not be of major importance for the disk chemistry of $\beta$ Pic. Alexander et al. (2004) studied the effects of X-ray ionization and heating on the stucture of CS disks around low-mass stars. Using parameters of typical T Tauri stars, they find that photoevaporation does not play a significant role in the evolution of $\mathrm{CS}$ disks. Given the fact that the X-ray flux from $\beta$ Pic is very low, we expect that this is also valid for $\beta$ Pic. 


\section{Conclusions}

We have detected X-ray emission of the A5 V star $\beta$ Pic in a $73 \mathrm{ks}$ observation with XMM-Newton. Despite the low number of photons, a thorough statistical analysis makes us confident that the observed excess in the OVII triplet is actually real with a flux level of $6.0 \times 10^{-16} \mathrm{erg} / \mathrm{cm}^{2} / \mathrm{s}$. We discuss the detected $\mathrm{X}$-ray emission in view of (i) the existence of a cool corona and (ii) accretion of circumstellar material in a boundary layer.

The main results of this work can be summarized as follows:

(1) $\beta$ Pic shows weak emission in the OVII triplet at $21.6 \AA$. We derive an energy flux of $6.0 \times 10^{-16} \mathrm{erg} / \mathrm{cm}^{2} / \mathrm{s}$ in the OVII triplet. To our knowledge this is the first detection of $\mathrm{X}$-rays from $\beta$ Pic.

(2) The OVII detection is consistent with a cool corona with $T \approx 600000 \mathrm{~K}$.

(3) $\beta$ Pic exhibits highly ionized species typical of a transition region (Deleuil et al. 2001). The emission from the CIII and OVI lines are significantly broadened compared to the otherwise similar star Altair.

(4) Material is accreted on $\beta$ Pic with (as measured for FEBs) velocities up to $400 \mathrm{~km} \mathrm{~s}^{-1}$. This might account for the additional broadening of the CIII and OVI emission lines.

(5) Accretion in a boundary layer appears to be capable of explaining the observed UV lines of CIII and OVI and the weakness of $\mathrm{O}$ VII.

Acknowledgements. This work is based on observations obtained with XMM-Newton, an ESA science mission with instruments and contributions directly funded by ESA Member States and the USA (NASA). We acknowledge support by the DLR under grants DLR 50 OR 0005 and 50 OR 0105. Finally, we thank the anonymous referee for constructive comments and suggestions to improve this paper.

\section{References}

Alexander, R. D., Clarke, C. J., \& Pringle, J. E. 2004, MNRAS, 354, 71

Ayres, T. R., Simon, T., Stern, R. A., et al. 1998, ApJ, 496, 428

Berghöfer, T. W., Schmitt, J. H. M. M., \& Hünsch, M. 1999, A\&A, 342, L17

Brandeker, A., Liseau, R., Olofsson, G., \& Fridlund, M. 2004, A\&A, 413,681

Bouret, J.-C., Deleuil, M., Lanz, T., et al. 2002, A\&A, 390, 1049

Chen, C. H., \& Kamp, I. 2004, ApJ, 602, 985

Christensen-Dalsgaard, J. 2000, ed. M. Breger, \& M. Montgomery, San Francisco, ASP Conf. Ser., 210, 187

Chung, S.M., Drake, J. J., Kashyap, V. L., et al. 2004, ApJ, 606, 1184

Deleuil, M., Bouret, J.-C., Lecavelier des Etangs, A., et al. 2001, ApJ, 557, L67
Dere, K. P., Landi, E., Mason, H. E., et al. 1997, A\&AS, 125, 149

Di Folco, E., Thévenin, F., Kervella, P., et al. 2004, A\&A, 426, 601

Ehle, M., Breitfellner, M., Gonzales Riestra, M., et al. 2003, XMM-Newton User's Handbook

Ferlet, R., Vidal-Madjar, A., \& Hobbs, L. M. 1987, A\&A, 185, 267

Freire Ferrero, R., Gouttebroze, P., Catalano, S., et al. 1995, ApJ, 439, 1011

Freytag, B., Ludwig, H. G., \& Steffen, M. 1996, A\&A, 313, 497

Gerbaldi, M., Zorec, J., Castelli, F., \& Faraggiana, R. 1993, ASP Conf., 44, 413

Golub, L., Harnden, F. R., Jr., Maxson, C. W., et al. 1983, ApJ, 271, 264

Holweger, H. 2001, in Solar and Galactic Composition, A Joint SOHO/ACE Workshop, ed. R. F. Wimmer-Schweingruber, AIP Conf. Proc., 598, 23

Holweger, H., Hempel, M., van Thiel, T., \& Kaufer, A. 1997, A\&A, 320, L49

Holweger, H., Hempel, M., \& Kamp, I. 1999, A\&A, 350, 603

Hempel, M., \& Schmitt, J. H. M. M. 2003, A\&A, 408, 971

Hünsch, M., Schmitt, J. H. M. M., Voges, W. 1998, A\&AS, 132, 155

Hünsch, M., Schmitt, J. H. M. M., Sterzik, M. F., \& Voges, W. 1999, A\&AS, 135, 319

Kamp, I., van Zadelhoff, G.-J., van Dishoeck, E. F., \& Stark, R. 2003, A\&A, 397, 1129

Kamp, I., \& Sammar, F. 2004, A\&A, 427, 561

King, J. R., \& Patten, B. M. 1992, MNRAS, 256, 571

Kürster, M., Schmitt, J. H. M. M., Cutispoto, G., \& Dennerl, K. 1997, A\&A, 320, 831

Kupka, F., \& Montgomery, M. H. 2002, MNRAS, 330, L6

Lagrange, A.-M., Beust, H., Mouillet, D., et al. 1998, A\&A, 330, 1091

Ness, J.-U., \& Wichmann, R. 2002, AN, 323, 129

Ness, J.-U., Güdel, M., Schmitt, J. H. M. M., et al. 2004, A\&A, 427, 667

Ness, J.-U., Schmitt, J. H. M. M., \& Robrade, J. 2004, A\&A, 414, L49

Ohanesyan, J. B. 1997, Astrophysics, 40, 237

Petterson, O. K. L., \& Tobin, W. 1999, MNRAS, 304, 733

Redfield, S., Linsky, J. L., Ake, T. B., et al. 2002, ApJ, 581, 626

Schmitt, J. H. M. M., Golub, L., Harnden, F. R., et al. 1985, ApJ, 290, 307

Schmitt, J. H. M. M., Micela, G., Sciortino, S., et al. 1990, ApJ, 351, 492

Schmitt, J. H. M. M., \& Kürster, M. 1993, Science, 262, 215

Schmitt, J. H. M. M., \& Liefke, C. 2004, A\&A, 417, 651

Schneider, G., Smith, B. A., Becklin, E. E., et al. 1999, ApJ, 513, L127

Simon, T., Ayres, T. R., Redfield, S., \& Linsky, J. L. 2002, ApJ, 579, 800

Simon, T., \& Landsman, W. B. 1997, ApJ, 483, 435

Smith, B. A., \& Terrile, R. J. 1984, Science, 226, 1421

Song, I., Caillault, J.-P., Barrado y Navascués, D., \& Stauffer, J. R. 2001, ApJ, 546, 352

White, F. M. 1991, Viscous Fluid Flow (McGraw-Hill International Editions) 